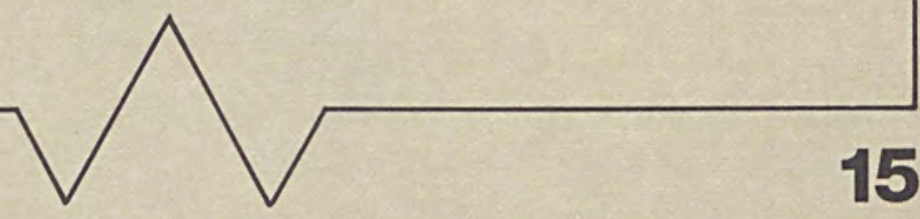

Hans Albert

Rechtswissenschaft als Realwissenschaft

Das Recht als soziale Tatsache und die Aufgabe der Jurisprudenz

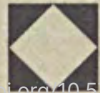

Nomos Verlag 
Würzburger Vorträge zur Rechtsphilosophie, Rechtstheorie und Rechtssoziologie

Herausgegeben von Hasso Hofmann, Edgar Michael Wenz und Dietmar Willoweit

Mitbegründet von Ulrich Weber

Heft 15 
Hans Albert

\section{Rechtswissenschaft als Realwissenschaft}

Das Recht als soziale Tatsache und die Aufgabe der Jurisprudenz 
Vortrag gehalten am 18. Februar 1993

Die Deutsche Bibliothek - CIP-Einheitsaufnahme

\section{Albert, Hans:}

Rechtswissenschaft als Realwissenschaft: Das Recht als soziale Tatsache und die Aufgabe der Jurisprudenz / Hans Albert. - 1. Aufl. - Baden-Baden: Nomos Verl.Ges., 1993

(Würzburger Vorträge zur Rechtsphilosophie, Rechtstheorie und Rechtssoziologie; H. 15) ISBN 3-7890-3130-5

NE: GT

$-952148-$

1. Auflage 1993

(C) Nomos Verlagsgesellschaft, Baden-Baden 1993. Printed in Germany. Alle Rechte, auch die des Nachdrucks von Auszügen, der photomechanischen Wiedergabe und der Übersetzung, vorbehalten. 


\section{Editorial}

Gerade angesichts unserer reich blühenden Dogmatik des positiven Rechts und im Hinblick auf eine gewisse Tendenz, Rechtspraxis auf Rechtstechnik zu reduzieren, ist es wichtig, ja notwendig, über den kleinen Kreis derjenigen hinaus, die sich mit Rechtsphilosophie, Normentheorie oder Rechtssoziologie besonders befassen, Anstöße für die Beschäftigung mit den Grund- und Grenzfragen des Rechts zu geben. Wenigstens ab und an sollte auch der vorwiegend dogmatisch arbeitende Jurist - Student wie Praktiker - jene heilsame Unruhe verspüren, die aus der Begegnung mit den Frag-Würdigkeiten der Grundlagen und Methoden unseres Faches entspringt. Eine lockere Folge von Einzelvorträgen scheint uns hierfür aus mehreren Gründen das geeignete Mittel. So kann auf diese Weise am ehesten ein lebendiger Eindruck von Reichtum, Vielfalt und Spannweite der nichtdogmatischen Beschäftigung mit dem Recht entstehen. Reichen die Grund- und Grenzprobleme unseres Faches doch von der alten und unabweisbar immer neu sich stellenden Frage der Gerechtigkeit, der Frage des Rechts als Ausdruck menschlichen Selbstverständnisses über die moderne Normanalytik bis zur Bedeutung der sozialen Verhältnisse für Entstehung, Anwendung und Wirksamkeit der Gesetze wie für die Bildung von Rechtsbewußtsein.

Durch die Veröffentlichung dieser an der Universität Würzburg gehaltenen Vorträge möchten die Herausgeber darüber hinaus Texte zur Verfügung stellen, die über bestimmte Aspekte überschaubare Zugänge zur Theorie eröffnen, aber auch als Arbeitsmittel in Seminaren und Übungen dienen können.

Würzburg, im Juli 1984

Hasso Hofmann Ulrich Weber Edgar Michael Wenz 


\section{Rechtswissenschaft als Realwissenschaft Das Recht als soziale Tatsache und die Aufgabe der Jurisprudenz}

I. Die Realwissenschaft vom Recht und das Problem des Charakters der Jurisprudenz

Mein Ausgangspunkt ist die These, daß die Wissenschaft das Recht als soziale Tatsache auffassen und analysieren kann und daß es infolgedessen auch in theoretisch gestützten Erklärungen vorkommen kann. Es kann also eine Rechtswissenschaft als Realwissenschaft geben, und zwar als eine Realwissenschaft üblicher Art. Damit ist eine theoretische Wissenschaft gemeint in dem Sinne, in dem in der modernen Wissenschaftslehre von theoretischen Realwissenschaften die Rede ist. Was ich meine, ist eine Wissenschaft im Sinne eines Naturalismus, in dem unter anderem das Kausalprinzip akzeptiert ist, so daß kausale Erklärungen angestrebt werden ${ }^{1}$.

Wenn das Recht eine soziale Tatsache, ein Tatbestand des sozialen Lebens, ist, dann muß diese Wissenschaft auch eine Sozialwissenschaft sein. Eines der wichtigsten Probleme der modernen Sozialwissenschaften ist seit ihrer Entstehung im 17. Jahrhundert - vor allem im Rahmen der philosophischen Auffassungen von Thomas Hobbes - das Problem der sozialen Ordnung. Man könnte es in folgender Weise formulieren: Wie ist soziale Ordnung möglich? oder: Was sind die Bedingungen der Möglichkeit sozialer Ordnung? Unter welchen Bedingungen ist damit zu rechnen, daß soziale Ordnung entsteht oder

1 Natürlich ist ein schwaches Kausalprinzip gemeint, das auch Erklärungen auf der Basis stochastischer Gesetze zuläßt. 
daß irgendeine Art sozialer Ordnung zustande kommt, denn es kann ja verschiedene Arten solcher Ordnungen geben, die sich in einigen Eigenschaften unterscheiden. Das Recht gehört nun offenbar zu den sozialen Phänomenen, die im Rahmen dieser Ordnungsproblematik zu behandeln sind. Schon bei Hobbes spielt es in diesem Zusammenhang eine zentrale Rolle.

Nun ist das Recht ohne Zweifel auch ein Gegenstand der Jurisprudenz, also der Rechtswissenschaft, wie sie an den juristischen Fakultäten betrieben wird. Und man kann die Frage stellen, ob man diese Jurisprudenz als eine Realwissenschaft der erwähnten Art auffassen darf, oder ob es irgendeine Beziehung dieser Jurisprudenz zu einer Realwissenschaft vom Recht gibt, die der von mir gegebenen Beschreibung entspricht. Mit dieser Frage gerät man sofort in die heutige Kontroverse über den Charakter der Jurisprudenz und damit gleichzeitig auch in die sozialwissenschaftliche Methodenkontroverse. Denn es scheint ja keineswegs selbstverständlich zu sein, daß man die Jurisprudenz als eine Wissenschaft der genannten Art auffassen kann, obwohl sie sich zweifellos auf das geltende Recht bezieht. Und wenn das nicht der Fall ist, scheint auch ihre Beziehung zu einer solchen Wissenschaft nicht von vornherein klar zu sein.

Man wird zunächst entgegengehalten bekommen, daß man es in der Jurisprudenz nicht mit einem Sein, sondern mit einem Sollen zu tun hat, daß es nicht um Tatsachen - jedenfalls nicht um Tatsachen üblicher Art, wie man sie in den Naturwissenschaften findet -, sondern um Normen geht, und daß man hier nicht nach Erklärungen - im Sinne der Naturwissenschaft strebt, sondern auf ein Verstehen aus ist. Kurz, man wird mit der Auffassung konfrontiert, daß man in diesem Problembereich vom Naturalismus abzugehen und eine Wissenschaft gänzlich anderer Art ins Auge zu fassen habe, die sich daher auch anderer Methoden bedienen müsse, als sie etwa in den Naturwissenschaften an der Tagesordnung seien.

Dieses Argument gewinnt noch an Plausibilität, wenn man 
sich mit der Funktion der Jurisprudenz beschäftigt, mit den Aufgaben, die sie im sozialen Leben zu erfüllen hat. Offenbar geht es bei der Anwendung dieser Disziplin im wesentlichen darum, adäquate Entscheidungen für die Praxis vorzubereiten. Und diese Entscheidungen müssen aus Normen oder jedenfalls im Zusammenhang mit Normen gewonnen werden, die man verstehen muß, um solche Entscheidungen treffen zu können. Die betreffenden Normen dürfen aber nicht einfach willkürlich angenommen werden. Sie sind vielmehr - jedenfalls in vielen oder den meisten Fällen - vorgegeben und müssen akzeptiert werden. Da sie im allgemeinen nicht in Frage zu stellen sind weder vom praktizierenden Juristen noch vom Vertreter der Rechtswissenschaft - sind sie meist gewissermaßen als Dogmen hinzunehmen. Damit ist hier keineswegs ein Dogmatismus im schlechten Sinne des Wortes gemeint, sondern es soll nur darauf hingewiesen werden, daß jede Willkür auszuschließen ist. Hypothesen kann es dann bestenfalls über die richtige Auslegung der Normen geben. Und das ist dann eine Angelegenheit der Hermeneutik als der Wissenschaft von der richtigen Auslegung oder einer hermeneutisch verfahrenden Jurisprudenz.

Im Gegensatz zu einer Realwissenschaft naturalistischen Typs wäre die Rechtswissenschaft dann eher eine dogmatische, normative und hermeneutische Disziplin in dem von mir charakterisierten Sinne. Sie hätte dogmatisch - das heißt in Dogmen - begründete normative Aussagen mit den Mitteln der Hermeneutik, also einer verstehenden Methode, zu finden.

Ich möchte ausdrücklich feststellen, daß das eine durchaus plausible - also keineswegs unsinnige - Auffassung ist, und weiter: daß in der Begründung dieser Auffassung, wie ich sie eben in aller Kürze dargestellt habe, viele Elemente enthalten sind, die ich für akzeptabel halte, obwohl ich die Konsequenzen, die daraus gezogen werden, nicht akzeptieren kann. Wir hätten hier jedenfalls eine plausible Alternative zur naturalistischen Auffassung der Rechtswissenschaft, von der ich ausge- 
gangen bin. Falls man auch eine solche Rechtswissenschaft naturalistischen Charakters zuließe - und ich glaube kaum, daß man ihre Möglichkeit ohne weiteres von vornherein ausschlieBen kann -, wäre allerdings noch die Frage zu klären, in welcher Beziehung die Jurisprudenz zu dieser Realwissenschaft vom Recht stünde. Es ist kaum anzunehmen, daß die beiden Disziplinen für einander völlig irrelevant sein könnten. $\mathrm{Zu}$ nächst möchte ich aber feststellen, was ich an der erwähnten Begründung für eine Jurisprudenz nicht naturalistischen Charakters für akzeptabel oder jedenfalls für bedenkenswert halte. Es handelt sich im wesentlichen um drei Punkte:

(1) Die Rechtswissenschaft muß den tatsächlich geltenden Normen Rechnung tragen. Sie kann also nicht selbst willkürlich Recht setzen. Das ist der richtige Kern der dogmatischen Auffassung, wenn man sie einmal so nennen will.

(2) Es geht in der Tat unter anderem um die Erfassung von normativen Regelungen sozialer Verhältnisse, also von Normen, wie das in der normativen Auffassung akzentuiert wird. Und:

(3) Es müssen sinnhafte Komponenten des sozialen $\mathrm{Ge}$ schehens erfaßt und daher unter anderem verstehende Methoden angewendet werden, wie das die hermeneutische Auffassung betont.

Die entscheidende Frage ist aber, ob das alles einen Gegensatz zum Naturalismus involviert. Ich bin der Ansicht, daß das nicht der Fall ist, und zwar aus folgenden Gründen:

(1) Wenn man den tatsächlich geltenden Normen gerecht werden will, dann muß das nicht zu einer dogmatischen Auffassung im angegebenen Sinne des Wortes führen. Es kann sich ja jeweils um Hypothesen über tatsächlich geltende oder auch über mögliche Normen handeln, ohne daß man genötigt wäre, diese Normen als maßgebend zu akzeptieren, mit ihnen also einen normativen Anspruch zu verbinden.

(2) Die Erfassung von Normen bedingt keineswegs ohne weiteres eine normative Wissenschaft. Es kann sich vielmehr 
um eine Realwissenschaft üblicher Art handeln, die sich mit Normen als Tatbeständen sozialer Steuerung befaß $\mathrm{t}^{2}$.

(3) Die Benutzung verstehender Methoden muß nicht zu einer Wissenschaft besonderer Art führen, wie sie manche Vertreter der Hermeneutik anstreben. Max Weber hat zum Beispiel verstehende Erklärungen angestrebt, das heißt: Kausalerklärungen unter Einbeziehung sinnhafter Komponenten des Geschehens, deren Erfassung die Anwendung verstehender Methoden voraussetzt.

Man kann sogar sagen, daß es zumindest eine Realwissenschaft gibt, in der alle diese Punkte beachtet werden, nämlich die Rechtssoziologie, wie sie etwa von Max Weber aufgefaßt wurde. Sie strebt nach verstehenden Erklärungen rechtlicher Phänomene unter Verwendung theoretischer Hypothesen und versucht, soziale Ordnungen zu erklären, zu deren Kern das geltende Recht gehört ${ }^{3}$.

Das mag wohl sein, wird man sagen. Aber darin liegt gerade der Unterschied zwischen der Rechtssoziologie und der Jurisprudenz. Die erste mag eine Realwissenschaft vom Recht im üblichen Sinne des Wortes sein. Unter Umständen ist man vermutlich sogar bereit, hier dem Naturalismus ein Zugeständnis zu machen. Aber die Jurisprudenz, so wird man fortfahren, ist eine praxisbezogene Wissenschaft vom Recht, die gar nicht die Aufgabe hat, Erklärungen dieser Art zu erreichen, sondern die Entscheidungen vorbereiten soll, wie ich das eben schon erwähnt habe. Darin genau liegt der Unterschied zwischen den Aufgaben der beiden Disziplinen, und deshalb muß die eine einen gänzlich anderen Charakter haben als die andere.

2 Vgl. dazu das III. Kapitel: Das Problem der sozialen Steuerung und die Idee einer rationalen Jurisprudenz, meines Buches: Traktat über rationale Praxis, Tübingen 1978, sowie Peter Behrens, Die ökonomischen Grundlagen des Rechts. Politische Ökonomie als rationale Jurisprudenz, Tübingen 1986.

3. Vgl. dazu neuerdings Michael Baurmann, Der Markt der Tugend. Recht und Moral in der liberalen Gesellschaft (erscheint in Kürze). 
Diesem Argument würde ich sofort zustimmen. Ich würde nur die Frage daran knüpfen, was daraus für den Charakter der Jurisprudenz folgt. Auch Wissenschaften naturalistischen Typs können ja zur Vorbereitung von Entscheidungen beitragen, und sie tun das in großem Maße. Wie machen sie das? Sie können eine solche Leistung erbringen, weil sie die Ableitung von technologischen Aussagen - von Technologien - gestatten.

Was sind Technologien? Technologische Aussagensysteme sind, kurz gesagt, Systeme von Aussagen über Handlungsmöglichkeiten, die aus theoretischen Aussagen über Wirkungszusammenhänge gewonnen werden können. Sie antworten auf die Frage, was getan werden kann oder muß, um bestimmte Ziele zu erreichen. Aussagen dieser Art sind durchaus zur Vorbereitung von Entscheidungen zu gebrauchen. Sie sagen dem Handelnden allerdings nicht, was er tun soll, sondern eben nur, was er tun kann oder was er tun muß, um bestimmte Ziele zu erreichen.

II. Normativismus und Sozialtechnologie: Zwei Deutungen der Jurisprudenz

Wir könnten demnach die Möglichkeit ins Auge fassen, daß die Jurisprudenz technologisch zu deuten ist. Ich weiß, daß das Wort "Technologie « in der Anwendung auf soziale Zusammenhänge unbeliebt ist, weil man es vielfach - wenn auch völlig willkürlich - mit "Technokratie" assoziiert. Aber wenn man von "Kunstlehre" spricht, was auf dasselbe hinausläuft, wird die Sache vielleicht akzeptabel. Man könnte also die Deutung der Jurisprudenz als einer Sozialtechnologie, einer Kunstlehre zur Vorbereitung sozialer Entscheidungen bestimmter Art, ins Auge fassen. Dann wäre die Beziehung einer theoretischen Realwissenschaft vom Recht zur Jurisprudenz auf einfache Weise 
geklärt. Die Jurisprudenz wäre das sozialtechnologische Pendant zu dieser theoretischen Wissenschaft.

Wir haben nun also zumindest zwei alternative Deutungen der Jurisprudenz, zwischen denen zu entscheiden wäre:

(1) eine Deutung, die aus der normativistischen Auffassung entspringt, und deren Beziehung zu den Realwissenschaften ungeklärt ist, und

(2) eine sozialtechnologische Deutung, die einen unmittelbaren Zusammenhang zur Realwissenschaft vom Recht herstellt.

Bei der einen, so könnte man meinen, sei die Jurisprudenz imstande, jeweils ein Sollen festzustellen, bei der anderen sei sie nur imstande, ein Können zu konstatieren. Es liegt nahe, die erste Variante vorzuziehen, weil sie die Ableitung der betreffenden Entscheidungen zu gestatten scheint.

Aber kann es eine normative Wissenschaft geben, das heißt eine Wissenschaft, die statt kognitiver Aussagen normative Aussagen produziert, also Aussagen, die nicht wahrheitsfähig sind, also weder wahr noch falsch sein können? Normalerweise wird man jeder Wissenschaft zumindest die Aufgabe zuweisen wollen, nach Erkenntnis zu streben, auch der Jurisprudenz. Als Vertreter der normativistischen Auffassung wird man also geneigt sein, die scharfe Unterscheidung zwischen kognitiven und normativen Aussagen zurückzuweisen, die ich oben getroffen habe. Gibt es aber so etwas wie normative Erkenntnis? Wer das annimmt, der muß wohl annehmen, daß normative Aussagen kognitiven Charakter haben und demnach wahrheitsfähig sind. Es gibt in der Tat philosophische Auffassungen, die so etwas behaupten, zum Beispiel die phänomenologische Wertphilosophie Max Schelers oder der Intuitionismus George Moores, der mit nicht-natürlichen Eigenschaften operiert. Diese Auffassungen haben, soweit ich sehe, der Kritik nicht standgehalten. Sie unterstellen eine unhaltbare Ontologie und postulieren Einsichtsarten, für deren Existenz keinerlei An- 
haltspunkte zu finden sind ${ }^{4}$. Ich beschränke mich hier auf diesen Hinweis und gehe nun davon aus, daß jeder Wert- oder Normen-Platonismus unakzeptabel ist.

Man könnte nun darauf hinweisen, daß die normativen Aussagen einer solchen Jurisprudenz nur logische Konsequenzen vorgegebener Normen seien und demnach aus normativen Aussagen ableitbar, deren Geltung vorausgesetzt werden müsse. Daher gehe es hier nur um die Richtigkeit logischer Ableitungen, nicht um eine kognitive Deutung normativer Aussagen. Dann bestünde aber das Geschäft der Jurisprudenz nur in der Erstellung logischer Ableitungen, was kaum akzeptabel sein dürfte. Von Hermeneutik könnte keine Rede mehr sein.

Jedenfalls müßte der Tatsache Rechnung getragen werden, daß in dieser Disziplin Deutungsvorschläge für normative Aussagen gemacht $\mathrm{zu}$ werden pflegen, und solche Vorschläge könnten wiederum nicht aus normativen Erkenntnissen stammen, weil es diese nicht gibt. Sie könnten aber auch nicht ohne weiteres aus Erkenntnissen über die tatsächlichen Zusammenhänge stammen, die mit Hilfe kognitiver - also wahrheitsfähiger - Aussagen formulierbar sind. Denn bekanntlich lassen sich aus solchen Aussagen über Tatsachen keine normativen Aussagen ableiten. Bei einer solchen Ableitung würde man einen naturalistischen Fehlschluß begehen.

Es bliebe nur der Ausweg, entweder den normativen Anspruch aufzugeben, der mit den betreffenden Deutungsvorschlägen verbunden ist, und die Aufgabe der Jurisprudenz auf eine rein hermeneutische Leistung zu reduzieren oder diesen normativen Anspruch auf andere Weise zu begründen. Den ersten Weg scheint die reine Rechtslehre zu gehen, auf die ich noch zurückkommen werde.

4 Vgl. z.B. die Kritik in J. L. Mackie, Ethik. Auf der Suche nach dem Richtigen und Falschen, Stuttgart 1981, Viktor Kraft, Grundlagen einer wissenschaftlichen Wertlehre, 2. Auflage, Wien 1951, sowie in Albert/Topitsch (Hg.), Werturteilsstreit, 2. Aufl. Darmstadt 1979. 
Nun soll sich aber die Jurisprudenz auch nach der normativistischen Auffassung wohl vor allem jeweils auf das in einem bestimmten Raum-Zeit-Gebiet faktisch geltende Recht beziehen und damit auch auf faktische Zusammenhänge, auf Tatsachen bestimmter Art. Sie soll das faktisch geltende Recht identifizieren und interpretieren, oder vielleicht besser: Sie soll mit Hilfe ihrer Deutungsvorschläge das faktisch geltende Recht identifizieren, damit auf dieser Grundlage adäquate Entscheidungen getroffen werden können, also Entscheidungen, die sich als adäquate Anwendungen der betreffenden Normen ausweisen lassen.

Damit haben wir nun den Begriff der "faktischen Geltung" eingeführt, und es entsteht die Frage nach dem Charakter dieses Begriffs. Sind mit seiner Hilfe bestimmte Tatsachen erfaßbar? Dann wären Aussagen über die faktische Geltung von Normen nichts anderes als Aussagen über soziale Tatsachen, aus denen keine normativen Konsequenzen gezogen werden könnten. Oder handelt es sich um einen normativen Begriff, so daß eine Aussage über die faktische Geltung bestimmter Normen eine normative Aussage wäre. Sie wäre aber wieder aus Erkenntnissen über tatsächliche Zusammenhänge nicht ableitbar. Die Bezugnahme auf das faktisch geltende Recht führt also unter Umständen zu Schwierigkeiten, die im Rahmen der normativistischen Auffassung kaum zu überwinden sind. Es sind die Schwierigkeiten einer »reinen Jurisprudenz«, das heißt einer Rechtswissenschaft, deren Beziehung zu den Tatsachen des sozialen Lebens problematisch ist ${ }^{5}$.

5 Vgl. meinen Aufsatz: Zur Kritik der reinen Jurisprudenz. Recht und Rechtswissenschaft in der Sicht des kritischen Rationalismus, in: Internationales Jahrbuch für Rechtsphilosophie und Gesetzgebung, 1992, S. 343-357, sowie meine anschlieBende Diskussion mit Robert Walter, S. 358-364. 
III. Die Rechtssoziologie und die verstehende Erklärung sozialer Tatsachen

Die Rechtssoziologie versteht sich offenbar im allgemeinen als eine Realwissenschaft üblicher Art. Sie behandelt das Recht also als eine soziale Tatsache und zwar als Teil der sozialen Ordnung. Es geht ihr um die Erklärung sozialer Ordnungen, in denen das Recht eine zentrale Rolle spielt ${ }^{6}$. Solche Erklärungen müssen, wie schon erwähnt, verstehende Erklärungen sein, in denen das Funktionieren solcher Ordnungen aus dem Zusammenspiel der Handlungen - und damit aus den Entscheidungen der betreffenden Personen, auch der in Frage kommenden Funktionäre, der Rechtsorgane, - erklärt wird. Dabei geht es vor allem um das Funktionieren rechtlicher Sanktionsmechanismen und damit auch um die Anwendung und Durchsetzung von Normen, die von den betreffenden Personen verstanden werden müssen, um in ihren Entscheidungen eine Rolle spielen zu können.

Entscheidend sind also letzten Endes die Annahmen über das Verhalten der betreffenden Individuen, der "Rollenträger" in den sozialen Gebilden, die für die Erklärung in Betracht kommen, also zum Beispiel der Richter, der Verwaltungsbeamten, der Polizisten, aber auch über das Verhalten aller anderen Individuen, die zum Geltungsbereich der betreffenden Ordnung gehören. Für ihre Entscheidungen ist unter anderem die jeweilige "Definition der Situation " maßgebend, das heißt die Art ihrer Situationswahrnehmung, die auch die jeweils relevanten Rechtsnormen enthalten muß, das heißt diejenigen Normen, die für die Orientierung ihres Handelns maßgebend sind. Sie gehören zu den wirksamen Sinnkomponenten dieses Handelns. Das heißt also, daß die Identifikation der betreffenden Normen und ihre Anwendung auf die betreffende Entschei-

6 Vgl. Theodor Geiger, Vorstudien zu einer Soziologie des Rechts, Kopenhagen 1947. 
dungssituation hier eine kausale Rolle spielen, kausale Relevanz haben muß. Wie ich schon erwähnt habe, hat schon Max Weber erkannt, daß man so sinnhaftes Verhalten kausal erklären kann.

Es gibt seit langem eine Diskussion über Gründe und Ursachen, in der manche Theoretiker die Unterscheidung dieser beiden Begriffe dazu benutzen, das Verstehen von Handlungen gegen kausale Erklärungen abzugrenzen, die angeblich für sinnhaftes - und daher verstehbares - Verhalten nicht in Frage kommen. Das ist eine meines Erachtens unhaltbare Position. Sinnhaftes Verhalten zeichnet sich eben dadurch aus, daß in ihm Gründe zu Ursachen werden, daß sie also im Motivationsgeschehen eine kausale Rolle erhalten ${ }^{7}$.

Das Motivationsgeschehen ist nach allem, was heute bekannt ist, eine sehr komplexe Angelegenheit, mit der sich Psychologen und Vertreter der Sozialwissenschaften mit mehr oder weniger großem Erfolg beschäftigen, soweit sie an der Erklärung von Handlungen interessiert sind. Neben kognitiven Faktoren spielen in ihm offenbar auch noch andere Faktoren eine Rolle, nämlich solche, die die Interessen der Handelnden betreffen. Dabei kommt natürlich auch ein durch normative Überzeugungen gestütztes Interesse an adäquaten Entscheidungen in Betracht, das übrigens unter bestimmten Bedingungen durchaus mit dem Eigeninteresse des Handelnden kompatibel sein, bzw. in sein Eigeninteresse aufgenommen sein kann, so daß der Handelnde in diesem Falle nicht nur deshalb normkonform handelt, weil er die mit den betreffenden Normen verbundene Sanktionsdrohung berücksichtigt ${ }^{8}$.

7 Man kann daher mit Recht von Motivkausalität sprechen, vgl. dazu schon Arthur Schopenhauer, Die vierfache Wurzel des Satzes vom zureichenden Grunde (1813), Hamburg 1957.

8 Vgl. Viktor Vanberg/Roger D. Congleton, Rationality, Morality, and Exit, American Political Science Review, Vol. 86, No. 2, 1992, Hartmut Kliemt, Normbegründung und Normbefolgung in Ethik und Ökonomik, Zeitschrift für Betriebswirtschaft, 1992, S. 91-105, und Michael Baurmann, Der Markt der Tugend, a.a.O. 
Wer also solche Entscheidungen erklären will, muß mit dem geltenden Recht vertraut sein, zum Beispiel weil er genötigt ist, die Entscheidungssituation eines Richters zu rekonstruieren, was unter Umständen eine äußerst komplexe Aufgabe ist. Was dabei dann herauskommt, ist eine verstehende Erklärung, wie sie im Rahmen eines recht verstandenen Naturalismus möglich ist, also eine kausale Erklärung, hinter der Gesetzmäßigkeiten der Willensbildung und des Handelns stehen, die prinzipiell psychologisch erforschbar sind. Man wird sich allerdings vielfach mit einer Erklärungsskizze begnügen müssen, in der die Interpretation der Situation mit Hilfe des relevanten Normensystems durch den Erklärenden an die Stelle einer Darstellung der Zusammenhänge nach dem üblichen Erklärungsschema tritt ${ }^{9}$. Wenn außerdem die Annahme eines Interesses an einer adäquaten Entscheidung berechtigt erscheint, das durch kein anderes Motiv konterkariert wird, darf man eine solche Erklärung oft als zureichend ansehen. Natürlich bedeutet die Orientierung des Handelns an Normen nicht ohne weiteres, daß auch normkonform gehandelt wird. Es können ja - auch bei den Rechtsorganen - Motive auftreten, die dafür sorgen, daß das geltende Recht verletzt wird. Offenbar spielt die Orientierung am geltenden Recht auch für die Situationswahrnehmung anderer Personen eine erhebliche Rolle, zum Beispiel für die eines Kaufmanns, der sich zwischen verschiedenen Gesellschaftsformen für sein Unternehmen zu entscheiden hat, wobei etwa ein Gewinninteresse oder ein Interesse an der Vermeidung bestimmter Risiken zu unterstellen wäre, um seine Entscheidung zu erklären.

9 Vgl. dazu meine Darstellung in meinem Aufsatz: Erkenntnis und Recht. Die Jurisprudenz im Lichte des Kritizismus, (1972), in: Gerd Roellecke, (Hg.), Rechtsphilosophie oder Rechtstheorie, Darmstadt 1988, S. 289-314, bes. S. 303-307. 


\section{Das Problem der faktischen Geltung von Normen}

Es ist hier vielleicht angebracht, auf das Problem der faktischen Geltung von Normen zurückzukommen, das jedenfalls auch in die Rechtssoziologie gehört. Es bietet sich zunächst an, den Begriff der faktischen Geltung so zu bestimmen, daß man an die Verhaltenswirksamkeit der betreffenden Normen anknüpft. Normen, die in der Bevölkerung eines bestimmten Raum-Zeit-Gebietes in ausreichendem Maße verhaltenswirksam sind, könnte man als "faktisch gültig " bezeichnen. Aber das wäre eine zu schwache Anforderung. Sie reicht schon deshalb nicht aus, weil auch eine Orientierung an Normen möglich ist, die mit der Verletzung der betreffenden Normen verbunden ist. Auch hier sind ja diese Normen in bestimmter Weise verhaltenswirksam. Um diese Möglichkeit zu berücksichtigen, könnte man die Normkonformität des Verhaltens der betreffenden Individuen fordern, aber das wäre offenbar eine $\mathrm{zu}$ starke Anforderung, da der Begriff der faktischen Geltung dann kaum noch anwendbar wäre. Faktische Geltung möchte man sicher auch einem System von Normen zusprechen, das in einer Gesellschaft soweit verankert ist, daß auftretende Normverletzungen, wenn sie erkannt werden, im allgemeinen im Einklang mit bestimmten Normen sanktioniert werden. Theodor Geiger hat daher den Vorschlag gemacht, die Wirklichkeit einer Norm - und damit das, was mit faktischer Geltung gemeint sein kann - als ihre "Alternativwirkungs-Chance « zu bestimmen $^{10}$, so daß sie zu einer meßbaren Größe wird und damit Abstufungen zuläßt. "Eine Norm", sagt er, "ist nicht schlechthin gültig oder geltungslos, sondern verbindlich in höherem oder geringerem Grad. Gewisse Normen werden strenger, andere minder konsequent befolgt und durchgesetzt«. Ge-

$10 \mathrm{Vgl}$. Theodor Geiger, Vorstudien zu einer Soziologie des Rechts, a.a.O., S. 34 ff. 
gen diesen Begriff faktischer Geltung gibt es aber schwerwiegende Einwände, auf die ich hier nicht eingehen kann ${ }^{11}$.

Als Ausweg bietet es sich an, für den Begriff der faktischen Geltung auf den Willen eines Normgebers zurückzugehen, so daß das Sollen in einem Wollen verankert ist. Es muß sich allerdings um ein in einem bestimmten Sinne verhaltenswirksames Wollen handeln, nämlich in dem Sinne, daß Normadressaten Gründe haben, den betreffenden Willen zu erfüllen ${ }^{12}$, wobei solche Gründe insofern relevant sind, als sie kausale Bedeutung für die Willensbildung der Normadressaten haben können. Die faktische Geltung von Normen in diesem Sinne ist demnach stets als eine soziale Tatsache anzusehen, so daß eine diesbezügliche Behauptung kognitiven Charakter hat und daher prinzipiell wahrheitsfähig ist, also wahr oder falsch sein kann.

Soweit sich die Jurisprudenz mit dem faktisch geltenden Recht beschäftigt, dürfte auch sie darauf angewiesen sein, auf diesen für die Rechtssoziologie maßgebenden Begriff faktischer Geltung zu rekurrieren. Diese These scheint mir den Kern des Gegensatzes zu enthalten, der zwischen meiner Auffassung und der Auffassung besteht, die mir Rudolf Thienel entgegengehalten hat ${ }^{13}$. "Geltung", in dem von ihm angeführten Sinne, so sagt er zum Beispiel an einer Stelle, sei "nichts Faktisches - nicht eine Seinstatsache -, sondern etwas ideelles, nämlich der Umstand, daß eine bestimmte Pflicht existier(t)e,

11 Vgl. dazu Michael Baurmann, Der Markt der Tugend, a.a.O., S. 60 ff. Unter anderem würde ein Sanktionsmodell der Normgeltung die Klasse der Ermächtigungsnormen nur unzureichend erfassen.

12 Vgl. dazu Norbert Hoerster, Zum Problem der absoluten Normgeltung, in: Heinz Mayer u.a. (Hg.), Staatsrecht in Theorie und Praxis. Festschrift für Robert Walter zum 60. Geburtstag, Wien 1991, S. 255 ff. und an Hoerster anknüpfend, Baurmann, a.a.O., S. 54-64.

$13 \mathrm{Vgl}$. dazu Rudolf Thienel, Kritischer Rationalismus und Jurisprudenz. Zugleich eine Kritik an Hans Alberts Konzept einer sozialtechnologischen Jurisprudenz, Wien 1991, S. 65 und passim. Vielem, was er in dieser interessanten und gründlichen Untersuchung feststellt, kann ich dagegen zustimmen. 
also ein Sollen ". Das ist für seine an der reinen Rechtslehre Hans Kelsens orientierte Auffassung charakteristisch, in der ein Reich ideeller Gebilde postuliert wird, das einer Realwissenschaft nicht zugänglich ist.

Es ist vielleicht angebracht, in der hier gebotenen Kürze auf die Argumente einzugehen, die von der reinen Rechtslehre gegen den Realismus vorgebracht zu werden pflegen. Zunächst sei betont, daß die Vertreter dieser Auffassung ebenso wie die Vertreter des Realismus sich ausdrücklich von der Benutzung eines metaphysischen Geltungsbegriffs distanzieren, da sie davon ausgehen, daß gültige Normen von Menschen geschaffen werden und ihre Geltung menschlicher Autorität verdanken. Nur behaupten die Vertreter der reinen Rechtslehre, man könne normativen Phänomenen nicht gerecht werden, wenn man auf spezifisch normative Betrachtungen verzichte. Sogar eine soziologische Analyse solcher Phänomene setze derartige Betrachtungen und daher auch eine Jurisprudenz normativ dogmatischen Charakters voraus, und zwar schon zur Abgrenzung ihres Gegenstandsbereichs. "Geltung" als "die spezifische Existenz einer Norm, ... . ihre Existenz im Bereich des Sollens", sei "sowohl von der Existenz des Willensaktes, dessen Sinn die Norm sei, als auch von deren Wirksamkeit, beides Seinstatsachen, zu unterscheiden ${ }^{14}$.

Nun ist offenbar der Sinn eines Willensaktes oder einer entsprechenden Disposition ${ }^{15}$, wie er zum Beispiel im Inhalt einer normativen Aussage zum Ausdruck kommt, ein Merkmal dieses Aktes oder dieser Disposition, das man zwar wie andere Merkmale einer isolierten Betrachtung unterziehen kann, das aber dadurch keineswegs eine selbständige Existenz gewinnen

14 Vgl. dazu Michael Schmidt, Reine Rechtslehre versus Rechtsrealismus, in: Robert Walter (Hg.), Schwerpunkte der Reinen Rechtslehre, Wien 1992, S. 145.

15 Einem Vorschlag Hoersters folgend, greift Baurmann - a.a.O., S. $58 \mathrm{f}$. - zur Bestimmung des Geltungsbegriffs statt auf den Willensakt auf eine entsprechende Disposition des Normgebers zurück. 
kann. Es ist natürlich möglich, die betreffende normative Aussage einer Analyse im Hinblick auf diesen Sinn zu unterziehen. Solche normativen Aussagen, wie sie zum Beispiel in Rechtstexten auftreten, sind aber keineswegs als Beschreibungen von Normen aufzufassen, also als Aussagen, die Normen zum Gegenstand haben, sondern als Formulierungen von Normen und damit als Aussagen präskriptiven Charakters. Das bedeutet unter anderem, daß sie nicht wahrheitsfähig sind - also nicht wahr oder falsch sein können -, sondern nur befolgt oder nicht befolgt werden können. In der Wissenschaft - und zwar sowohl in der Rechtssoziologie als auch in der Jurisprudenz - gehören sie zum Gegenstandsbereich der Analyse, das heißt: es wird über sie gesprochen. Wenn man hier von Geltung spricht, dann kann das zunächst nichts anderes bedeuten, als daß man einen Anspruch auf allgemeine Anerkennung konstatiert, der mit diesen Regeln verbunden zu sein pflegt, der also faktisch erhoben und mehr oder minder erfolgreich durchgesetzt werden kann.

Wenn aber, wie das in der reinen Rechtslehre der Fall ist, von der "spezifischen Existenz einer Norm" die Rede ist, dann erweckt das unnötigerweise den Eindruck, es gebe einen Bereich außerhalb der Realität des sozialen Lebens, in dem Normen als Gegenstände besonderer Art existieren und auf den sich dann eine deskriptiv verfahrende Spezialdisziplin beziehen könne, die dennoch mit normativen Aussagen operiere. Gäbe es diesen Bereich und hätten die betreffenden Aussagen tatsächlich beschreibenden Charakter, dann müßte man ihnen auch einen Wahrheitswert zuschreiben können. Dann wäre es aber unerfindlich, inwiefern sie gleichzeitig etwas vorschreiben könnten. Vielleicht kann ein einfaches Beispiel die Pointe meines Arguments anschaulich machen. Man stelle sich vor, ein Offizier gebe den Befehl: "Ganze Batterie Feuer!«, der durch seine Autorität gedeckt und insofern gültig sei. Man könnte nun den Inhalt dieser Aussage, also den Sinn des darin enthaltenen Befehls, isolieren und die Existenz einer Norm behaupten, die einem entsprechenden ideellen Bereich angehöre, ẹi- 
nem Bereich, der von der Realität zu unterscheiden sei. Ich glaube kaum, daß man geneigt wäre, einer solchen Behauptung zuzustimmen. Und die Idee, die Kriegsgeschichte setze schon für ihre Abgrenzung eine entsprechende normative Disziplin voraus, würde sicher kaum akzeptiert werden.

Man kann meines Erachtens dem normativen Charakter bestimmter Texte durchaus gerecht weden, ohne Annahmen dieser Art zu machen. Auch die Deutung des subjektiven Sinnes von Handlungen im Weberschen Sinne setzt keine derartigen Annahmen voraus. Überdies sind sie schon deshalb höchst problematisch, weil sie nicht nur die Jurisprudenz von dem Bereich sozialer Tatsachen lösen würden, auf den sie sich letzten Endes doch beziehen soll. Es wäre darüber hinaus unerfindlich, in welcher Beziehung der Kosmos der Normen zur Rechtswirklichkeit stehen würde, mit der sich zum Beispiel die Rechtssoziologie zu befassen hat. In dieser Hinsicht ist jedenfalls hier kein wesentlicher Unterschied zum oben kritisierten Normenplatonismus zu erkennen. Die Vertreter der reinen Rechtslehre sind, wie schon erwähnt, gerade in dieser Hinsicht gegenteiliger Meinung.

Was die Kelsensche Auffassung angeht, in der Rechtswissenschaft müsse man zu Erkenntniszwecken jeweils eine Grundnorm voraussetzen, weil das für die Begründung der objektiven Geltung der positiven Rechtsnormen unerläßlich $\operatorname{sei}^{16}$ - sie sei gewissermaßen die transzendental-logische Voraussetzung der Rechtserkenntnis -, so beruht diese Auffassung selbst auf der Annahme, die Jurisprudenz müsse die von ihr erkannten Normen als objektiv gültig behandeln. Auch wenn es sich dabei "um eine reine Konstruktion ohne jede metaphysische Implikation handelt ${ }^{17}$, so daß man nicht den Einwand machen kann, daß hier ein Rückfall in naturrechtliches Denken 
vorliegt, ist nicht einzusehen, daß man in der Jurisprudenz eine solche Annahme benötigt. Denn man kann sich hier mit dem oben erörterten Begriff der faktischen Geltung begnügen.

Die Rechtssoziologie benutzt den Begriff faktischer Geltung jedenfalls im Rahmen eines Erkenntnisprogramms, das auf die Erklärung sozialer Tatsachen abzielt. Die Jurisprudenz stellt sich offenbar andere Aufgaben. Auf die Frage des Zusammenhangs zwischen den beiden Disziplinen werde ich noch zurückkommen.

V. Rationale Jurisprudenz als Grundlage von Gesetzgebung, Rechtsprechung und Verwaltung

Ganz unabhängig von der Frage, wie die an den juristischen Fakultäten betriebene Jurisprudenz aufzufassen ist, läßt sich die Frage nach der Möglichkeit einer sozialtechnologischen Disziplin untersuchen, die sich mit dem Problem der sozialen Ordnung befaßt, also auch mit dem Recht als zentralem Bestandteil der sozialen Ordnung in einer modernen Gesellschaft ${ }^{18}$. Eine solche technologische Disziplin hat, wie schon erwähnt, die Erkenntnisse der betreffenden theoretischen und historischen Realwissenschaften über soziale Wirkungszusammenhänge zu verwerten, und aus ihnen Aussagen über menschliche Handlungsmöglichkeiten zu gewinnen, die als Grundlage für Entscheidungen dienen können. Natürlich lassen sich die betreffenden Entscheidungen nicht aus solchen Aussagen logisch ableiten. Dazu benötigt man weitere Prämissen, die sich

18 Die Möglichkeit einer solchen Disziplin wird von Vertretern der reinen Rechtslehre eingeräumt. Nur wird darauf hingewiesen, daß sie eine Rechtsdogmatik im Kelsenschen Sinne voraussetzt; vgl. Robert Walter, Bemerkungen zu Albert, Zur Kritik der reinen Jurisprudenz. Recht und Rechtswissenschaft in der Sicht des kritischen Rationalismus, in: Internationales Jahrbuch für Rechtsphilosophie und Gesetzgebung, 1992, S. 359-362; vgl. auch Rudolf Thienel, a.a.O., S. 212 ff. 
vor allem auf die jeweiligen Zielsetzungen und auf die Zulässigkeit von Mitteln beziehen. Das heißt also, daß man die betreffenden Handlungsmöglichkeiten unter bestimmten praktischen Gesichtspunkten bewerten muß, um sie in Entscheidungen umzusetzen.

Es hat in der Sozialphilosophie und in den Sozialwissenschaften seit dem 17. Jahrhundert eine ganze Reihe von Beiträgen zur Lösung sozialtechnologischer Probleme gegeben, die sich auf die Ermöglichung sozialer Ordnung beziehen. Vor allem in der schottischen Moralphilosophie und in der politischen Ökonomie, die sich unter ihrem Einfluß entwickelt hat, sind solche Beiträge zu finden. Die zentrale Fragestellung ist, grob gesagt: Wie läßt sich soziale Ordnung erreichen? oder genauer: Wie läßt sich eine soziale Ordnung erreichen, die bestimmten Adäquatheitsbedingungen genügt, das heißt: die gewisse Eigenschaften oder Kombinationen von Eigenschaften besitzt? Im Anschluß an Rutledge Vining möchte ich hier von "Leistungsmerkmalen" sprechen ${ }^{19}$. Wie ist also eine Ordnung mit bestimmten Leistungsmerkmalen realisierbar? Unter Umständen ist es praktisch interessant, alternative realisierbare Ordnungen vergleichend zu untersuchen, um festzustellen, in welchem Ausmaß sie verschiedene Leistungsmerkmale besitzen, wie Freiheit oder verschiedene Arten von Freiheit, soziale Sicherheit, und verschiedene Grade von Stabilität oder Flexibilität. Die betreffenden Leistungsmerkmale können so ausgewählt sein, daß anzunehmen ist, ihre Realisierung liege im Interesse der Betroffenen, also der Mitglieder der betreffenden Gesellschaft, etwa ihrer Sicherung gegen Gewalt, gegen Willkür, gegen Ausbeutung, Armut, kurz gegen soziale Übel bestimmter Art.

$19 \mathrm{Vgl}$. Rutledge Vining, Economics in the United States of America, Paris 1956 , S. 10-14 wo von "performance characteristics « die Rede ist. 
Diese Fragestellung kann insofern praktische Bedeutung haben, als sie sich auf eine mögliche Gesetzgebung beziehen läßt, mit der die Absicht verbunden ist, eine derartige Ordnung herbeizuführen. Es geht dann darum, welche Gesetze einzuführen wären, um eine Ordnung zu erzeugen, die bestimmte Leistungsmerkmale besitzt. Offenbar sind die betreffenden Normen hier nicht als Selbstzweck zu betrachten, sondern nur als Mittel, um bestimmte Wirkungen zu erzielen ${ }^{20}$, wobei die erstrebten Wirkungen zuweilen unmittelbar verständlich erscheinen, wie bei manchen Normen des Strafrechts, manchmal aber für ihr Verständnis spezielle Kenntnisse voraussetzen, weil es um Wirkungen geht, die durch soziale Mechanismen vermittelt sind. Das letztere ist zum Beispiel bei den Normen der Fall, die die Bundesbank ermächtigen, etwa die Mindestreservesätze der Banken oder den Diskontsatz zu erhöhen, um eine Vermehrung der Geldmenge zu verhindern, die zur Inflation beitragen würde. Das Ziel, die Verhinderung inflationärer Entwicklungen, muß hier in sehr indirekter Weise erreicht werden, nämlich über komplizierte Kausalketten, die in Rechnung zu stellen sind und deren Berücksichtigung geldtheoretische Überlegungen erfordert. In einer modernen Gesellschaft zielt ein großer Teil der durch Gesetzgebung etablierten Normen auf solche indirekten Wirkungen und setzt daher theoretische Überlegungen dieser Art voraus, die natürlich richtig oder falsch sein können, man denke zum Beispiel an das Wohnungsrecht oder an die Gesetzgebung im Agrarbereich. In diesen und anderen Bereichen pflegen Ökonomen nicht selten auf den

20 Das gilt auch für fundamentale Normen, wie Hoerster mit Recht feststellt: „Normative Maßstäbe, oberste Normen (des Rechts und der Moral) sind nicht vom Menschen in einer metaphysischen Realität vorgefundene Wahrheiten sondern von Menschen erfundene oder geschaffene Instrumente sozialen Zusammenlebens « vgl. Norbert Hoerster, Verteidigung des Rechtspositivismus (Würzburger Vorträge zur Rechtsphilosophie, Rechtstheorie und Rechtssoziologie, hg. v. Ulrich Weber, Hasso Hofmann und Edgar Michael Wenz, Heft 11), Frankfurt am Main 1989. S. 28. 
kontraproduktiven Charakter gesetzgeberischer Maßnahmen hinzuweisen.

Das bedeutet aber unter anderem, daß eine Wissenschaft, die auf eine mögliche Gesetzgebung bezogen ist, also eine rationale Jurisprudenz, wie ich sie einmal nennen will, nicht im Sinne des erwähnten Normativismus aufgefaßt werden kann. Sie kann nicht einfach Systeme von Normen formulieren, die sich auf die jeweils erwünschten Merkmale sozialer Zustände beziehen, um aus ihnen logische Konsequenzen abzuleiten. Die Wirkungen, die mit Hilfe der betreffenden Gesetzgebung erzielt werden können, ergeben sich nämlich nicht als logische Konsequenzen aus den betreffenden Normen. Die Feststellung der betreffenden Wirkungszusammenhänge setzt vielmehr theoretische Überlegungen über die ihnen zugrundeliegenden Gesetzmäßigkeiten voraus. Daher kann eine solche Jurisprudenz nur sozialtechnologisch im erwähnten Sinne dieses Wortes aufgefaßt werden. Sie behandelt die Normsetzung als Mittel, um Wirkungen zu erzielen, die im Interesse von Mitgliedern der betreffenden Gesellschaft zu liegen scheinen. Um das $\mathrm{zu}$ erreichen, ist es notwendig, bestimmte Erkenntnisse über tatsächliche Zusammenhänge zu verwerten. Auch die Herstellung rechtsstaatlicher Verhältnisse in einem bestimmten Raum-Zeit-Gebiet ist zunächst ein sozialtechnologisches und dann natürlich auch ein praktisch-politisches Problem. Die Zähmung der Herrschaft durch das Recht läßt sich nicht ohne die Kenntnis der relevanten Wirkungszusammenhänge erreichen.

Wer die Gesetzgebung unter dem Gesichtspunkt betrachtet, daß mit ihrer Hilfe soziale Probleme in rationaler Weise gelöst werden sollen, wird Gesetze als Mittel zur Realisierung bestimmter Ziele behandeln und daher ihre voraussichtlichen Wirkungen berücksichtigen müssen. Jede Gesetzgebung ist unter dieser Voraussetzung daher auf sozialtechnologisches Wissen der von mir skizzierten Art angewiesen, gleichgültig, ob ihr dieses Wissen von einer wissenschaftlichen Disziplin geliefert 
wird oder nicht, das heißt, ob sie genötigt ist, sich auf das vorhandene Alltagswissen zu verlassen. Eine für die Gesetzgebung brauchbare Wissenschaft wird jedenfalls den Charakter einer rationalen Jurisprudenz in dem von mir skizzierten Sinne haben müssen ${ }^{21}$.

Jede wirksame Gesetzgebung läuft ja auf eine Umsteuerung des sozialen Geschehens hinaus, und damit auf eine - wenn auch noch so geringe - Umgestaltung der sozialen Ordnung. Sie wird dadurch erreicht, daß die Anreize für menschliches Verhalten in einer Weise verändert werden, die derartige Wirkungen zur Folge hat. Das gilt natürlich auch für die Einführung von Verfassungen oder für verfassungsändernde Maßnahmen, aus denen sich Programme für die Gesetzgebung ergeben können. Alle solchen Projekte bedürfen einer sozialtechnologischen Fundierung, wenn man daran interessiert ist, sie wirksam umzusetzen. Soweit der Bereich der Gesetzgebung in Betracht gezogen wird, ist also gegen eine Jurisprudenz dieser Art kaum etwas einzuwenden.

Hinsichtlich der Rechtsprechung und der Verwaltung scheint es zunächst anders auszusehen, da es hier vor allem um die Anwendung vorhandener Gesetze geht. Aber ist das wirklich der Fall? Gibt es hier einen wesentlichen Unterschied, der eine andere Art von Jurisprudenz notwendig machen würde, etwa die Jurisprudenz, die ich eingangs als Alternative zur sozialtechnologischen geschildert habe? Führt hier das prakti-

$21 \mathrm{Vgl}$. dazu das III. Kapitel meines Buches: Traktat über rationale Praxis, a.a.O., bes. S. 81 ff. Zur Möglichkeit einer technologischen Behandlung der Ordnungsproblematik vgl. auch Hartmut Kliemt, Das Denken in Ordnungen und die Möglichkeiten ordnungspolitischen Handelns, in: Ordnung in Freiheit, Tübingen 1992, S. 31-59. Was immer man gegen Jeremy Benthams utilitaristische Auffassung und gegen seinen darauf gegründeten Optimismus einwenden mag, man wird schwerlich etwas gegen die von ihm vertretene Ansicht einwenden können, daß eine Kunstlehre von der Gesetzgebung als sozialtechnologische Disziplin an theoretische Erkenntnisse über menschliches Verhalten anknüpfen müsse, wie es auch hier angenommen wird, vgl. dazu Jeremias Benthams, des englischen Juristen, Prinzipien der Gesetzgebung, hg. v. Etienne Dumont, Köln 1833. 
sche Erkenntnisinteresse, das für die Jurisprudenz geltend gemacht werden kann, zu anderen methodologischen Konsequenzen als für den Bereich der Gesetzgebung, so daß man hier eine dogmatische, normative und hermeneutische Disziplin benötigt, wie ich sie oben erwähnt habe?

Zunächst ist zuzugestehen, daß es hier um die Identifizierung und Anwendung der Normen des geltenden Rechts geht, wobei die Interpretation der in dieser Hinsicht relevanten Texte - der Gesetzestexte, Kommentare und Entscheidungssammlungen - eine erhebliche Rolle spielt. Die Jurisprudenz hätte also vor allem adäquate Deutungsvorschläge bereitzustellen, Vorschläge, die geeignet sind, etwa richterliche oder administrative Entscheidungen zu fundieren. Nun pflegen aber die relevanten Texte einen gewissen Deutungsspielraum offen $\mathrm{zu}$ lassen; und überdies scheint es vielfach nötig zu sein, das Normensystem des geltenden Rechts durch bestimmte Auslegungsverfahren zu ergänzen, um bestehende Lücken zu schließen. Das heißt aber, daß die Rechtsordnung bis zu einem gewissen Grade durch richterliche Entscheidungen mitgestaltet wird, so daß die Feststellung der geltenden Ordnung selbst so etwas wie eine schöpferische Leistung involviert.

Die hier verlangten hermeneutischen Bemühungen scheinen also einen ganz besonderen Charakter zu haben, der sie von denen wesentlich unterscheidet, die etwa in der Historiographie verlangt werden. Vertreter des Normativismus pflegen in diesem Zusammenhang den teleologischen Gesichtspunkt ins Spiel zu bringen. Sie weisen nämlich auf die Zwecke hin, an denen sich die Jurisprudenz bei ihren Deutungsvorschlägen zu orientieren habe $\mathrm{h}^{22}$. Da Normen rationalerweise nicht als Selbstzweck zu behandeln sind, ist es auch dann sinnvoll, für ihre Deutung von einer solchen Zwecksetzung auszugehen,

$22 \mathrm{Vgl}$. dazu Norberto Bobbio, Über den Begriff der "Natur der Sache«, in: Arthur Kaufmann (Hg.), Die ontologische Begründung des Rechts, Darmstadt 1965 , S. $87-103$. 
wenn dieser Zweck nicht offenkundig und daher nicht leicht eruierbar ist. Sobald man aber für die betreffenden Deutungsvorschläge auf Zwecke zurückgreift, muß man sich mit möglichen Wirkungen der zu identifizierenden Normen befassen. Damit gerät man eo ipso in den Bereich des technologischen Denkens. Es geht nämlich darum, die kausale Relevanz der zu identifizierenden Normen für die Herstellung der in Frage kommenden Ordnung zu berücksichtigen, einer Ordnung, die bestimmten Anforderungen genügt, also bestimmte Leistungsmerkmale aufweist.

Auch hier werden also die betreffenden Normen als Mittel betrachtet, mit denen bestimmte Wirkungen auf das Verhalten der Mitglieder der Gesellschaft zu erzielen sind, als Instrumente sozialer Steuerung. Die in Frage kommenden Deutungsvorschläge werden also im Hinblick auf diese zu erzielenden Wirkungen formuliert, so daß hier ebenfalls Erkenntnisse über soziale Wirkungszusammenhänge verwertet werden müssen. $\mathrm{Da} ß$ die betreffenden Deutungen in den Kontext des faktisch geltenden Rechts - also der übrigen Normen des betreffenden Systems - passen müssen, ändert nichts an dieser Tatsache.

Wenn man in diesem Zusammenhange von Hermeneutik redet, muß man sich also darüber klar sein, daß es sich um eine sehr spezielle Art von Hermeneutik handelt. Wer nämlich den Sinn des Rechts - den Sinn faktischer und möglicher Rechtsnormen und auch den Sinn von Texten, in denen solche Normen formuliert sind - bestimmen möchte, der sollte sich darüber klar sein, daß er sich dabei mit den Wirkungen zu beschäftigen hat, die mit ihnen angestrebt werden und damit also mit der Ordnung des sozialen Lebens, deren Realisierung beabsichtigt ist. Es geht also mehr um teleologischen als um semasiologischen Sinn. Dabei ist offenbar eine technologische Verwertung nomologischen Wissens erforderlich, denn, wie ich schon erwähnt habe, sind die Steuerungswirkungen von Normen keineswegs mit den logischen Konsequenzen der betreffenden normativen Aussagen identisch. 
Die Hermeneutik, die hier benötigt wird, ist also de facto eine sozialtechnologische Disziplin, die auf in einem bestimmten Sinne wirksame Deutungsvorschläge abzielt, ebenso wie die auf eine mögliche Gesetzgebung bezogene Disziplin auf in einem bestimmten Sinne wirksame Normen abzielt. Demnach hätte eine rationale Jurisprudenz dieser Art zwei Teile, einen Teil, der die Identifikation der Normen des - jeweils in einem bestimmten Raum-Zeit-Gebiet - geltenden Rechts durch effiziente Interpretationen ermöglicht, und einen Teil, der die Konstruktion effizienter Normen zur Modifikation des geltenden Rechts durch Gesetzgebung anstrebt ${ }^{23}$. Dazu bedarf es in beiden Fällen keiner normativen Aussagen. Es werden vielmehr nur Aussagen benötigt, in denen die betreffenden Normen als wirksam im Sinne hypothetisch vorausgesetzter Zwecke ausgezeichnet werden. Die Aussagen, in denen dies geschieht, sind wahrheitsfähig und als Hypothesen prinzipiell revidierbar. Und soweit verstehende Methoden für ihr Zustandekommen in Betracht kommen, sind diese im oben erwähnten Sinne aufzufassen, der kausale Deutungen involviert. Und die Beziehung dieser Art von Jurisprudenz zur theoretischen Realwissenschaft vom Recht ist unproblematisch. Sie ist eine technologische Disziplin, die im üblichen Sinne Ergebnisse dieser Realwissenschaft verwerten kann, soweit solche vorliegen. Soweit sie nicht vorliegen, muß die Praxis, wie das auch in anderen Bereichen geschieht, sich mit der Verwertung des alltäglichen Wissens begnügen, wie das wohl auch weitgehend der Fall ist.

Nach meinem Eindruck wird diese Auffassung über den Charakter der Jurisprudenz den zu Anfang erörterten plausiblen Elementen der Gründe gerecht, die für eine hermeneutische Auffassung der Jurisprudenz im traditionellen Sinne zu

23 Wer den Begriff der Effizienz als problematisch ansieht, sei daran erinnert, daß die Wirkung von Normen und Interpretationen sich stets auf die angestrebte soziale Ordnung bezieht, die bestimmte Leistungsmerkmale besitzt. 
sprechen scheinen. Sie nimmt außerdem die brauchbaren Elemente der Interessenjurisprudenz in sich auf und wird den Einwänden gerecht, die gegen die Begriffsjurisprudenz erhoben wurden ${ }^{24}$. Auch der Vorwurf, diese Auffassung involviere eine Selbstbeschränkung der Rechtswissenschaft, der sich teilweise auf Thesen der jüngeren Frankfurter Schule stützt, kann, wie ich zu zeigen versucht habe ${ }^{25}$, als auf Mißverständnissen beruhend zurückgewiesen werden.

VI. Rechtswissenschaft und Rechtspraxis im Rechtsstaat: Die Rolle der Jurisprudenz in der Gestaltung des sozialen Lebens

Daß die Jurisprudenz der Rechtspraxis zu dienen hat, wird im allgemeinen kaum bestritten werden. Die Frage ist nur, wie sie ihre praktische Funktion am besten erfüllen kann. Die Jurisprudenz, von der hier die Rede ist, ist ein Resultat der europäischen Rechtsentwicklung, ebenso wie der Rechts- und Verfassungsstaat der Neuzeit, der, welthistorisch gesehen, einen Ausnahmefall darstellt. Er ist auch theoretisch ein ganz unwahrscheinlicher Fall, dessen Zustandekommen offenbar spezifische Bedingungen voraussetzt, wie sie in der europäischen Geschichte gegeben waren. Die Entstehung des Rechtsstaates ist ein Resultat des europäischen Sonderweges - des »europäischen Wunders ${ }^{26}$, also ein Produkt der europäischen Kul-

24 Vgl. dazu meinen Aufsatz: Zur Kritik der reinen Jurisprudenz, a.a.O., S. 349-353.

$25 \mathrm{Vgl}$. a.a.O., S. 352 f. $\mathrm{Zu}$ den Auffassungen von Habermas und Apel, die auch im juristischen Denken immer wieder als Grundlage für kritische Argumente ins Feld geführt werden, vgl. jetzt Herbert Keuth, Erkenntnis oder Entscheidung? Zur Kritik der kritischen Theorie Tübingen 1993, weiter Eric Hilgendorf, Argumentation in der Jurisprudenz, Berlin 1992, und auch die diesbezüglichen Abschnitte, in: Thienel, Kritischer Rationalismus und Jurisprudenz, a.a.O., S. 138-158.

26 Vgl. Eric Lionel Jones, The European Miracle, sec. edition, Cambridge 1987, deutsche Ausgabe: Das Wunder Europa, Tübingen 1991. 
$\operatorname{tur}^{27}$, das nur unter Schwierigkeiten exportiert werden kann, obwohl die weltweite Diskussion über Menschenrechte darauf hinweist, daß es überall starke Interessen in dieser Richtung gibt. Auch innerhalb Europas ist der Rechtsstaat, wie wir wissen, bis in die neueste Zeit immer wieder gefährdet gewesen. Vor allem im Rahmen einer rechtsstaatlichen Ordnung kann die Jurisprudenz als unabhängige Wissenschaft die Bedeutung haben, die ich ihr zugeschrieben habe.

Die historische Bedeutung des Rechtsstaates besteht vor allem darin, daß durch ihn bis zu einem gewissen Grade die Zähmung der Herrschaft gelungen ist, so daß eine Art von sozialer Ordnung möglich wurde, in der bürgerliche und politische Freiheit gesichert sind. Wie Berman gezeigt hat, knüpft die europäische Rechtsentwicklung seit der sogenannten päpstlichen Revolution des 11. und 12. Jahrhunderts zwar an das römische Recht an, geht aber in wesentlichen Punkten über das hinaus, was dort zu finden war. Es gibt in anderen Kulturen offenbar kein Recht als einen autonomen Bereich des sozialen Lebens, der durch die Konkurrenz verschiedener Rechtssysteme, durch professionelle Verwaltung, wissenschaftliche Bearbeitung und den Vorrang vor politischen Autoritäten charakterisiert ist und der sich unter dem Einfluß bestimmter Ideale entwickelt hat ${ }^{28}$. Der Konstitutionalismus, der die Bindung der Politik und damit auch die der Ausübung von Herrschaft an das Recht involviert, gehört offenbar von Anfang an zu den Idealen, die diese Entwicklung beeinflußt und die transnationale europäische Rechtskultur geprägt haben. Und die europäische Jurisprudenz, die die Entwicklung des Rechts konstruktiv und kritisch begleitet hat, war von Anfang an ein wichtiger Teil

27 Vgl. Harold Berman, Law and Revolution. The Formation of the Western Legal Tradition, Cambridge, Mass. 1983, wo unter anderem die Besonderheiten des europäischen Rechts und die Eigenart der europäischen Jurisprudenz herausgearbeitet werden.

$28 \mathrm{Vgl}$. Berman, a.a.O., S. 7 ff. 
dieser Rechtskultur ${ }^{29}$ und damit ein wichtiger Faktor für die Entwicklung des Konstitutionalismus, der dann in die moderne europäische Sozialphilosophie eingegangen ist, in der das Recht vor allem als Mittel der Sicherung des Friedens und der Freiheit aufgefaßt wurde.

Wie kann die Jurisprudenz nun im Rahmen dieser Rechtskultur ihre praktische Funktion am besten erfüllen? Nach üblicher Auffassung kann sie selbst keine rechtlich relevanten Entscheidungen treffen. Die Übernahme der von ihr erarbeiteten Problemlösungen in das geltende Recht ist Sache der dazu autorisierten Rollenträger der Praxis - der Gesetzgebungsgremien, der Richter und der administrativen Organe. Sie können ihre Entscheidungen im Lichte der von der Jurisprudenz gelieferten Erkenntnisse treffen. Das bedeutet aber, daß diese Disziplin eine wichtige Funktion für die Bestimmung des faktisch geltenden Rechts und damit auch für die Gestaltung der sozialen Ordnung hat, die ja in erheblichem Maße durch dieses Recht mitbestimmt ist. Zu den wichtigsten Aufgaben einer rationalen Jurisprudenz gehört meines Erachtens daher die Analyse des Problems einer sozialen Ordnung, die bestimmten Adäquatheitsbedingungen genügt, mit sozialtechnologischen Mitteln.

Die soziale Ordnung ist aber kein statisches Phänomen. Sie ist vielmehr in dauernder Veränderung begriffen, da laufend neuartige Probleme auftauchen, die bewältigt werden müssen. Die angemessene Bewältigung dieser Probleme setzt nicht nur

29 Vgl. Berman, a.a.O., S. 120-164. "Die neue Rechtsmethodologie, die im späten elften und im zwölften Jahrhundert im Westen entstand - ihre Logik, ihre Themen, ihr Argumentationsstil, ihr Niveau der Generalisierung, ihre Techniken der Verbindung von Besonderem und Allgemeinem, von Fällen und Begriffen - war*, wie Berman betont, "ein essentieller Teil der bewußten Systematisierung des Rechts als einer autonomen Wissenschaft. Und diese wiederum war ein essentieller Teil der Schöpfung eines autonomen Rechtssystems für die neuen politischen Formen, die aus der päpstlichen Revolution hervorgingen«, a.a.O., S. 163 f. (Übers. von mir. d.V.). 
Erkenntnisse über Tatsachen und über kausale Zusammenhänge voraus, sondern auch die Beachtung relevanter Wertgesichtspunkte für die Selektion brauchbarer Lösungen. Das Recht entwickelt sich nicht nur auf Grund von Erkenntnissen faktischer Zusammenhänge, sondern auch unter dem Einfluß von Interessen und Idealen. Auch eine technologisch aufgefaßte Jurisprudenz muß, wenn sie praktische Bedeutung haben will, solchen Wertgesichtspunkten Rechnung tragen. Sie kann das ohne Schwierigkeiten tun, indem sie das, was ich das Sachgerüst der Wertungen nennen möchte, explizit in ihren Untersuchungen berücksichtigt. Das ist möglich, indem sie die Leistungsmerkmale alternativer Problemlösungen herausarbeitet. Da solche Leistungsmerkmale faktische Eigenschaften sind, wird die Wahrheitsfähigkeit ihrer Aussagen nicht dadurch beeinträchtigt.

Wenn man diese Deutung der Jurisprudenz akzeptiert, dann hat man sich für eine Auffassung entschieden, derzufolge eine Kontinuität besteht zwischen den rationalen Grundlagen der Gesetzgebung - einschließlich der Verfassungsgesetzgebung -, der Rechtsprechung und der Verwaltung, denn alle Teile dieser Jurisprudenz beziehen sich auf eine anzustrebende adäquate Ordnung des sozialen Lebens, die unter dem Einfluß praktischer Entscheidungen in dauernder Entwicklung begriffen ist. Die Adäquatheitsbedingungen dieser Ordnung selbst sind Gegenstand einer öffentlichen Diskussion, an der sich natürlich auch die Vertreter der Rechtswissenschaft beteiligen. Sie können wie die Vertreter anderer Disziplinen auf Grund ihrer Fachkompetenz zur Aufklärung der Bürger über Zusammenhänge beitragen, die für die Lösung der Probleme sozialer Ordnung wichtig sind. Und sie können diese Kompetenz darüber hinaus auch kritisch einsetzen, indem sie etwa zu zeigen suchen, daß und inwiefern die Normen des geltenden Rechts oder die Normen, die in Gesetzgebungsprogrammen für die Änderung des geltenden Rechts vorgesehen sind, ungeeignet sind, zur Realisierung oder zur Stabilisierung einer im Sinne 
bestimmter Wertgesichtspunkte adäquaten Ordnung beizutragen. Schließlich können sie natürlich auch wie jeder an der Diskussion beteiligte Bürger auf die ungenügende Berücksichtigung bestimmter Leistungsmerkmale in den vorherrschenden Ordnungsidealen hinweisen. Allerdings wäre es wünschenswert, in jedem Falle deutlich zu machen, zu welcher Art von Problemen man sich äußert und damit auch, inwieweit man sich dabei auf Erkenntnisse stützt und inwieweit man Wertgesichtspunkte in die Diskussion einführt. Das Max Webersche Prinzip der Wertfreiheit hat keineswegs, wie vielfach angenommen wird, zur Folge, daß bestimmte Probleme aus der Diskussion ausgeschlossen werden. Es soll uns nur davon abhalten, Wertungen als Erkenntnisse auszugeben. 


\section{Hans Albert}

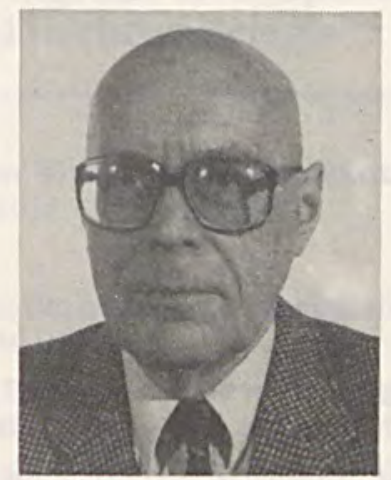

Geb.:

1939:

8. 2. 1921 in Köln am Rhein

1939-45:

Abitur

Mai 1945 bis

Dezember 1945:

Arbeitsdienst und Wehrdienst

1946-52:

amerikanische Kriegsgefangenschaft

Studium der Wirtschafts- und Sozialwissenschaften an der Universităt Köln

1950:

1952-58:

Diplom-Kaufmann, 1952 Dr. rer. pol.

1957:

Assistent am Forschungsinstitut für Sozial- und Verwaltungswissenschaften an der Universität Köln

Privat-Dozent an der Wirtschafts- und Sozialwissenschaftlichen Fakultät der Universität Köln

1958-63: $\quad$ Dozent an der Universität Köln

1963-89: ord. Professor für Soziologie und Wissenschaftslehre an der Universität Mannheim

Buchveröffentlichungen: Ökonomische Ideologie und politische Theorie, Göttingen 1954, 2. Aufl. 1972; Marktsoziologie und Entscheidungslogik, Neuwied/Berlin 1967; Traktat über kritische Vernunft, Tübingen 1958, 5. erw. Aufl. 1991; Plädoyer für kritischen Rationalismus, München 1971, 4. Aufl. 1975; Konstruktion und Kritik, Hamburg 1972, 2. Aufl. 1975; Theologische Holzwege. Gerhard Ebeling und der rechte Gebrauch der Vernunft, Tübingen 1973; Transzendentale Träumereien. Karl-Otto Apels Sprachspiele und sein hermeneutischer Gott, Hamburg 1975; Aufklärung und Steuerung, Hamburg 1976; Kritische Vernunft und menschliche Praxis, Stuttgart 1977, 2. Aufl. 1984 (Reclam); Traktat über rationale Praxis, Tübingen 1978; Das Elend der Theologie. Kritische Auseinandersetzung mit Hans Küng, Hamburg 1979; Die Wissenschaft und die Fehlbarkeit der Vernunft, Tübingen 1982; Freiheit und Ordnung, Tübingen 1986; Kritik der reinen Erkenntnislehre, Tübingen 1987; (mit Adorno/Dahrendorf/Habermas/Pilot/Popper), Der Positivismusstreit in der deutschen Soziologie, Neuwied/Berlin 1969, 13. Aufl. 1989. 


\section{Würzburger Vorträge zur Rechtsphilosophie, Rechtstheorie und Rechtssoziologie}

Heft 1: Arthur Kaufmann: Theorie der Gerechtigkeit, Problemgeschichtliche Betrachtungen, 1984, 51 S., 19,80 DM

Heft 2:-

Heft 3: Niklas Luhmann: Die soziologische Beobachtung des Rechts, 1986,48 S., $16,80 \mathrm{DM}$

Heft 4: Ernst-Wolfgang Böckenförde: Die verfassunggebende Gewalt des Volkes - Ein Grenzbegriff des Verfassungsrechts, 1986, 34 S., 16,80 DM

Heft 5: Ralf Dreier: Rechtsbegriff und Rechtsidee, Kants Rechtsbegriff und seine Bedeutung für die gegenwärtige Diskussion, 1986, 37 S., 18,80 DM

Heft 6: Günter Dux: Der Täter hinter dem Tun, Zur soziologischen Kritik der Schuld, 1988, 58 S., 24,- DM

Heft 7: Franz Bydlinski: Recht, Methode und Jurisprudenz, 1987, 46 S., 19,80 DM

Heft 8: Martin Kriele: Freiheit und „Befreiung“, Gibt es eine Rangordnung der Menschenrechte?, 1988. 52 S., 26,-DM

Heft 9: Manfred Rehbinder: Fortschritte und Entwicklungstendenzen einer Soziologie der Justiz, 1989, 63 S., 26,- DM

Heft 10: Klaus Lüderssen: Die Krise des öffentlichen Strafanspruchs, 1989, 62 S., 26,- DM

Heft 11: Norbert Hoerster: Verteidigung des Rechtspositivismus, 1989, 31 S., 26,- DM

Heft 12: Guiseppe Duso: Der Begriff der Repräsentation bei Hegel und das moderne Problem der politischen Einheit, 1990, 55 S., 24,- DM

Heft 13: Otfried Höffe: Gerechtigkeit als Tausch? Zum politischen Projekt der Moderne, 1991, 37 S., 18,- DM

Heft 14: Klaus F. Röhl: Die Gerechtigkeitstheorie des Aristoteles aus der Sicht sozialpsychologischer Gerechtigkeitsforschung, 1992, 59 S., 26,- DM 
